\title{
BMJ Open Fatigue, quality of life and metabolic changes in men treated with first-line enzalutamide versus abiraterone plus prednisolone for metastatic castration- resistant prostate cancer (HEAT): a randomised trial protocol
}

Klara Kvorning Ternov, ${ }^{\oplus}$ Jens Sønksen, ${ }^{1}$ Mikkel Fode, ${ }^{1}$ Henriette Lindberg, ${ }^{2}$ Caroline Michaela Kistorp, ${ }^{3}$ Rasmus Bisbjerg, ${ }^{1}$ Ganesh Palapattu, ${ }^{4}$ Peter Busch Østergren ${ }^{1}$

To cite: Kvorning Ternov K, Sønksen J, Fode M, et al. Fatigue, quality of life and metabolic changes in men treated with first-line enzalutamide versus abiraterone plus prednisolone for metastatic castration-resistant prostate cancer (HEAT): a randomised trial protocol. BMJ Open 2019;9:e030218. doi:10.1136/ bmjopen-2019-030218

- Prepublication history for this paper is available online. To view these files, please visit the journal online (http://dx.doi. org/10.1136/bmjopen-2019030218).

Received 08 March 2019 Revised 07 August 2019 Accepted 13 August 2019
Check for updates

(c) Author(s) (or their employer(s)) 2019. Re-use permitted under CC BY-NC. No commercial re-use. See rights and permissions. Published by BMJ.

For numbered affiliations see end of article.

\section{Correspondence to} Dr Klara Kvorning Ternov; klara.kvorning.ternov@regionh. $\mathrm{dk}$

\section{ABSTRACT}

Introduction Enzalutamide and abiraterone acetate plus prednisolone (AAP) are used in combination with androgen-deprivation therapy to further suppress the androgen stimulation of metastatic castration-resistant prostate cancer (mCRPC). First-line mCRPC treatment with enzalutamide and AAP yields similar overall survival and radiographic progression-free survival in phase III trials. Thus, treatment selection relies on patient choice, cost and side effects. The aim of this randomised trial is to investigate differences in fatigue, health-related quality of life (HRQoL) and metabolic side effects in men with $\mathrm{MCRPC}$ treated with first-line enzalutamide versus AAP.

Methods and analysis In this ongoing open-label randomised (1:1) clinical trial, enzalutamide is compared with AAP as first-line treatment for men with mCRPC. The primary endpoint is fatigue assessed with the questionnaire Functional Assessment of Chronic Illness Therapy-Fatigue version 4. Secondary endpoints are changes in body composition (ie, fat mass, visceral adipose tissue, subcutaneous adipose tissue and lean body mass assessed with dual energy X-ray absorptiometry), glucose metabolism assessed with a 2-hour oral glucose tolerance test, serum lipids, blood pressure and HRQOL assessed with the questionnaire Functional Assessment of Cancer Therapy-Prostate (FACT-P). All study endpoints are assessed at baseline and 12-week postintervention. Blood and urine samples are collected at baseline and at time of progression on allocated treatment for future investigation of predictive and prognostic biomarkers in prostate cancer treatment. The planned sample size is 170 participants. All participants are recruited from Herlev and Gentofte Hospital, Denmark. Estimated last patient's last visit is February 2020.

Ethics and dissemination The study received project approval from the National Committee on Health Research Ethics and Danish Data Protection Agency and Danish Medicines Agency (EudraCT no.: 2017-000027-99). The results of the study will be published in peer-reviewed

\section{Strengths and limitations of this study}

- This randomised clinical trial will report patient-reported and metabolic side effects in a relatively large sample size.

- This is the first randomised head-to-head trial primarily comparing fatigue, health-related quality of life and metabolic side effects in men with metastatic castration-resistant prostate cancer treated with first-line enzalutamide versus abiraterone plus prednisolone.

The trial lacks assessment of long-term side effects.

international journals and will be presented at national and international conferences and symposiums.

Trial registration number Clinicaltrialsregister.eu (2017000099-27).

\section{INTRODUCTION}

During the past decade, several new treatment options have emerged for metastatic castration-resistant prostate cancer (mCRPC). These new treatment options include the androgen pathway inhibitors enzalutamide and abiraterone acetate. Enzalutamide blocks several steps in the androgen receptor signalling. Abiraterone inhibits enzymes (17a-hydroxylase and 17,20-lyase) in the androgen biosynthesis, and is combined with prednisolone to compensate for abiraterone-induced reductions in serum cortisol. ${ }^{12}$

Enzalutamide and abiraterone acetate plus prednisolone (AAP) yield similar radiographic progression-free and overall survival results in the phase III trials (PREVAIL and COU-AA-302, respectively) which has led to 
approval as first-line mCRPC treatments. ${ }^{3-6}$ Thus, the choice between these two agents depends on the patient's preference, costs and agent-specific side effects.

Men with mCRPC that have been treated with androgen-deprivation therapy (ADT), have an increased risk of metabolic side effects and fatigue affecting health-related quality of life (HRQoL) ${ }^{78}$ Androgen pathway inhibitors are generally safe, with low rates of grade 3 and 4 adverse events. Most of the reported adverse events of these treatments are similar in the mentioned phase III trails, but the following adverse events were different: enzalutamide was associated with memory impairment and seizures; whereas AAP was associated with liver function abnormalities, peripheral oedema and cardiac events. ${ }^{359}$ Fatigue was the most common reported adverse event of both enzalutamide and AAP, although commonly emphasised as a side effect to enzalutamide. ${ }^{35}$ Comparing the results of the two trials is difficult, as the men in the control group of the enzalutamide trial (PREVAIL) received placebo, whereas the men in the control group of the AAP trial (COU-AA-302) received placebo plus prednisone. ${ }^{35}$ To date, no randomised head-to-head comparison primarily exploring differences in the side effect profiles have, to our knowledge, been published.

In addition, AAP has been approved for hormonenaive metastatic prostate cancer and enzalutamide is expected to gain similar approval ${ }^{10-12}$ (NCT02677896, NCT02319837). This change in treatment sequencing will result in longer exposure to side effects, making comparative studies with specific side effect endpoints even more essential.

This protocol describes an ongoing randomised clinical trial comparing self-reported fatigue and HRQoL and metabolic changes in men treated with enzalutamide or AAP as first-line treatment for mCRPC. The aim is that the results from this trial may help patients and physicians to choose the best tolerated treatment based on the difference in the side effects of enzalutamide and AAP.

\section{METHODS AND ANALYSIS}

\section{Trial design}

This is a single-centre open-labelled randomised (1:1) phase IV trial comparing first-line enzalutamide versus AAP in men with mCRPC. The trial is conducted at the Department of Urology, Herlev and Gentofte Hospital, Denmark. The primary objective is to compare fatigue assessed with the questionnaire Functional Assessment of Chronic Illness Therapy-Fatigue (FACIT-Fatigue) version 4.

\section{Participants}

Eligible participants are men with newly diagnosed mCRPC, defined as metastatic prostate cancer progressing on ADT, based on the prostate cancer working group 3 criteria. ${ }^{13}$ Metastatic status is measured with computed tomography (CT) scan of the thorax and abdomen and bone imaging $\left({ }^{18} \mathrm{~F}\right.$-sodium fluoride positron emission tomography-CT (PET/CT) or prostate-specific membrane antigen PET/CT). Inclusion criteria are age $\leq 90$ years, an Eastern Cooperative Oncology Group performance status $0-1$ and adequate organ function (creatinine $<1.5 \mathrm{x}$ the upper limit of normal (ULN), total bilirubin $<1.5 \times$ ULN and alanine aminotransferase or aspartate aminotransferase $\leq 2.5 \times$ ULN). Exclusion criteria are visceral metastases, a prior history of seizures, known heart failure (New York Heart Association functional class $>2$ ), diabetes mellitus, hypersensitivity to or previous treatment with enzalutamide or AAP or previous treatment with docetaxel. An exception to the latter is docetaxel in the metastatic hormone-naive prostate cancer setting, if the treatment was completed $>6$ months prior to enrolment. Inclusion and exclusion criteria are depicted in table 1. Eligibility is primarily assessed at the department's multidisciplinary team conference, where all patients are evaluated prior to starting first-line mCRPC treatment. Subsequently, eligibility is confirmed by the primary investigator at a screening consultation before randomisation. This ensures that all eligible men at Herlev and Gentofte Hospital are offered study participation. The timeline from screening to intervention is depicted in figure 1.

\section{Randomisation}

Patients who meet the inclusion and exclusion criteria and have given a written informed consent are randomised at the screening consultation using the Randomization Module of Research electronical data capture (REDCap V.7.1.1). The allocation sequence is a computer-generated list of random numbers transferred to REDCap by a collaborator with no clinical involvement in the trial. Participants are randomly assigned to either enzalutamide or AAP in a 1:1 ratio without stratification. Randomisation follows a block randomisation with 60 men in the first block and 110 men in the last block. Participants and physicians are aware of the allocation arm after randomisation. Data and outcome assessors are not blinded.

\section{Interventions}

Recruitment began in June 2017 and planned completion is December 2019. Herlev and Gentofte Hospital provides urological cancer care for a population of $\sim 1.3$ million. We estimate that around 150 men are offered either enzalutamide or AAP as first-line mCRPC treatment yearly at the Department of Urology, Herlev and Gentofte Hospital. Participants are randomised to receive one of the following treatments.

\section{Enzalutamide}

Participants are allocated to take $160 \mathrm{mg}$ enzalutamide orally in the evening.

\section{Abiraterone acetate plus prednisolone}

Participants are allocated to take $1000 \mathrm{mg}$ abiraterone acetate orally at least 1 hour before a meal or 2 hours after a meal in the evening. 
Table 1 Inclusion and exclusion criteria

\begin{tabular}{|c|c|}
\hline Inclusion criteria & Exclusion criteria \\
\hline $\begin{array}{l}\text { Eligible for first line treatment with either enzalutamide or } \\
\text { abiraterone acetate plus prednisolone as per standard of care } \\
\text { guidelines. } \\
\text { Age 18-90 years. } \\
\text { Willing, capable and legally competent individuals. } \\
\text { ECOG performance status } 0-1 \text {. } \\
\text { Histologically confirmed adenocarcinoma of the prostate. } \\
\text { Prior surgical orchiectomy or if on LHRH agonist/antagonist, } \\
\text { then testosterone }<1.7 \text { nmol/L at screening visit (participants } \\
\text { must maintain LHRH agonist/antagonist therapy for duration of } \\
\text { study treatment if not surgically castrated). } \\
\text { Evidence of metastatic disease on bone imaging or CT scan. } \\
\text { Evidence of biochemical or imaging progression in the setting } \\
\text { of surgical or medical castration. Progressive disease for } \\
\text { study entry is defined by one of the following criteria based on } \\
\text { criteria of PCWG3: } \\
\text { - Biochemical progression: obtain sequence of rising PSA } \\
\text { values at a minimum of } 1 \text { week intervals, resulting in } \\
\text { increases over the nadir, with PSA }>1 \text { ng/mL. } \\
\text { - Radiological progression: } \\
\text { - The appearance of two or more new bone lesions on } \\
\quad \text { bone imaging. } \\
\text { - Enlargement of a soft tissue lesion using the modified } \\
\text { RECIST V.1.1. } \\
\text { - Creatinine }<1.5 \times \text { ULN. } \\
\text { - Total bilirubin }<1.5 \times \text { ULN. } \\
\text { - ALT or AST } \leq 2.5 \times \text { ULN. }\end{array}$ & $\begin{array}{l}\text { Inability to understand and/or stick to the written information. } \\
\text { Previous treatment with docetaxel, with the exception of } \\
\text { previous treatment with early docetaxel ( } \leq 6 \text { series) } \geq 6 \text { months } \\
\text { before inclusion. } \\
\text { Diagnosed with diabetes mellitus and/or HbA1C }>48 \mathrm{mmol} / \mathrm{mol} \text {. } \\
\text { Hypersensitivity towards components in abiraterone acetate } \\
\text { plus prednisolone or enzalutamide. } \\
\text { Ongoing treatment with high doses of glucocorticoids. } \\
\text { Severe concurrent illness or comorbid disease that would make } \\
\text { the subject unsuitable for enrolment. } \\
\text { Prior therapy with CYP17 inhibitors, enzalutamide or other } \\
\text { experimental antiandrogens. } \\
\text { Life expectancy }<6 \text { months. } \\
\text { Active concurrent malignancy. } \\
\text { Treatment with Radium-223. } \\
\text { Known brain metastases. } \\
\text { Liver or lung metastases on CT scanning. } \\
\text { History of seizure or seizure disorder, or history of } \\
\text { cerebrovascular stroke within } 6 \text { months of study entry. } \\
\text { Known cardiac failure ( }>\text { NYHA class II). }\end{array}$ \\
\hline
\end{tabular}

ALT, alanine aminotransferase; AST, aspartate aminotransferase; CYP17, 17a-hydroxylase and 17, 20-lyase; ECOG, Eastern Cooperative Oncology Group; HbA1C, glycated haemoglobin; LHRH, luteinising hormone-releasing hormone; NYHA, New York Heart Association; PCWG3, Prostate Cancer Working Group 3; PSA, prostate-specific antigen; RECIST, Response Evaluation Criteria in Solid Tumours; ULN, upper limit of normal.

Participants are instructed to take $10 \mathrm{mg}$ prednisolone orally in the morning.

Compliance is ensured by registering the number of returned tablets at the follow-up visit. During the trial, all participants continue ADT. In addition, all participants follow normal standard of care and monitoring according

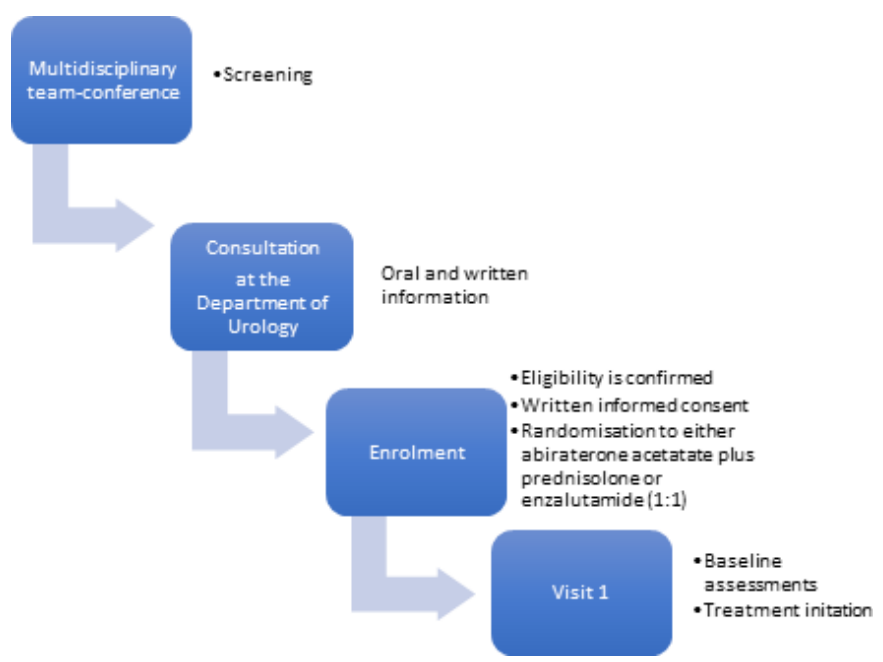

Figure 1 Timeline from screening to intervention. to local and national guidelines at the Department of Urology, Herlev and Gentofte Hospital, such as being offered bone protecting agents (ie, denosumab and calcium and vitamin D supplements). Participants receive allocated treatment from baseline visit until biochemical and/or radiographic progression or at the treating physician's discretion after which appropriate choice of second-line mCRPC treatment will be decided at multidisciplinary team conferences as per standard of care.

\section{Outcomes}

\section{Measurements}

Primary and secondary outcomes are assessed for all participants at baseline and 12-week post-intervention by the primary investigator. A schedule of enrolment, interventions and assessments is depicted in table 2.

\section{Primary outcome}

The primary endpoint is the between-group differences in changed level of fatigue assessed with the 13-question questionnaire FACIT-Fatigue available and validated in Danish. The participants report the past week's experienced fatigue by grading each question from one to four: 'not at all', 'a little bit', 'some-what', 'quite a bite' and 'very much'. 
Table 2 Schedule of enrolment, interventions and assessments

\begin{tabular}{|lllll|}
\hline Visit & $\begin{array}{l}\text { Randomisation } \\
\text { and enrolment }\end{array}$ & $\begin{array}{l}\text { Baseline } \\
\text { visit }\end{array}$ & $\begin{array}{l}\text { 12-week } \\
\text { follow-up }\end{array}$ & $\begin{array}{l}\text { Follow-up at time of } \\
\text { disease progression }\end{array}$ \\
\hline $\begin{array}{l}\text { Time (weeks from treatment } \\
\text { initiation) }\end{array}$ & $-2 \pm 2$ & 0 & $+12 \pm 2$ & $\begin{array}{l}+10 \text { until the year } \\
2023\end{array}$ \\
$\quad$ Written informed consent & $X$ & & & \\
Medical history & $X$ & $X$ & & \\
Medication list & $X$ & $X$ & $X$ & \\
\hline ECOG performance status & $X$ & $X$ & $X$ & \\
\hline
\end{tabular}

Physical

$\begin{array}{llll}\text { Height } & \text { X } & & \\ \text { Blood pressure, weight, BMI } & \text { X } & \text { X } & \\ \text { Questionnaires } & & & \text { X } \\ \text { FACIT-Fatigue } & \text { X } & \text { X } & \text { X } \\ \text { FACT-P } & \text { X } & \text { X } & \end{array}$

Samples

\begin{tabular}{|c|c|c|c|}
\hline Blood samples & $\mathrm{x}$ & $x$ & $x$ \\
\hline Urine sample & $\mathrm{x}$ & & $x$ \\
\hline \multicolumn{4}{|c|}{ Other paraclinical examinations } \\
\hline DXA scan & $x$ & $x$ & \\
\hline $\begin{array}{l}\text { OGTT (only the first } 60 \\
\text { participants) }\end{array}$ & $x$ & $x$ & \\
\hline \multicolumn{4}{|l|}{ Safety } \\
\hline Adverse events & & $\mathrm{x}$ & \\
\hline
\end{tabular}

BMI, body mass index; DXA, dual energy X-ray absorptiometry; ECOG, Eastern Cooperative Oncology Group; FACIT-Fatigue, Functional Assessment of Chronic Illness Therapy-Fatigue; FACT-P, Functional Assessment of Cancer Therapy-Prostate; OGTT, oral glucose tolerance test.

A minimal clinical important difference (MCID) in fatigue is defined as a 3.0 points change on an individual level. ${ }^{14}$ Fatigue is assessed at baseline, 12-week postintervention and at time of disease progression on allocated treatment.

\section{Secondary outcomes}

The secondary outcomes are the between-group differences in changed HRQoL, body composition, blood pressure, insulin sensitivity and resistance measured with a 4-point oral glucose tolerance test (OGTT), serum lipids and androgen treatment response.

\section{Health-related quality of life}

The between-group change in HRQoL is assessed with the 39-question questionnaire Functional Assessment of Cancer Therapy-Prostate (FACT-P) version 4 available and validated in Danish. FACT-P is assessed with the same grading as the FACIT-Fatigue questionnaire. An MCID in HRQoL is defined as a six points change on an individual level. ${ }^{15}$ HRQoL is assessed at baseline, 12 -week postintervention and at time of disease progression on allocated treatment.

\section{Body composition}

Fat mass, body fat $\%$, visceral adipose tissue volume (VAT), subcutaneous adipose tissue volume (SAT) and lean body mass (LBM) are obtained using DXA whole body fan-beam scans (Hologic Discovery, Bedford, Massachusetts, USA) with the software APEX V.4.0. VAT and SAT are measured in a $5 \mathrm{~cm}$ wide horizontal slice across the abdomen from the iliac crest to the L4-L5 segment. ${ }^{16} 17$ Weight (BWB-800A, TANITA, Tokoyo, Japan) and body mass index (height in meters ${ }^{2} /$ weight in kilograms) is assessed as well. Systolic and diastolic blood pressure is measured on the right arm after at least $20 \mathrm{~min}$ of rest (BP A3 Plus, Microlife AG, Widnau, Switerland).

\section{Oral glucose tolerance test}

The first 60 participants undergo a 2-hour OGTT (75 g glucose) after at least 9 hours of fasting. Plasma glucose and insulin are measured after 0,30, 60 and $120 \mathrm{~min}$. The whole body insulin sensitivity index (Matsuda index) are calculated from plasma glucose and insulin concentrations attained from the 2-hour OGTT in the subgroup of 60 participants. ${ }^{18}$ The equation for calculating the Matsuda index is: $10.000 / \sqrt{ }($ FPG $\times$ FPI $\times$ mean PG $\times$ mean PI). FPG is the fasting plasma glucose and FPI is the fasting plasma insulin concentration. Fasting plasma glucose and insulin are measured in all 170 participants. Fasting insulin resistance is calculated from the basal glucose and insulin concentrations using the 
homeostatic model assessment with following equation: $(\mathrm{FPG} \times \mathrm{FPI}) / 22.5{ }^{19}$

\section{Biochemical assays}

All blood samples are drawn before 11 am after a minimum of 9 hours of fasting.

\section{Metabolic analyses}

Plasma glucose is analysed with an enzymatic assay (Vitros 5.1, Ortho-Clinical Diagnostics, USA). Plasma insulin is analysed with a enzyme immunoassay (DRG instruments $\mathrm{GmbH}$, Germany). Glycated haemoglobin (HbAlc) is measured by high-performance liquid chromatography (Variant II TURBO, Bio-RAD, USA). Triglycerides, total cholesterol, high density lipoprotein cholesterol, low density lipoprotein cholesterol and very low density lipoprotein cholesterol are assayed by an enzymatic technique (Vitros 5.1 FS, Ortho-Clinical Diagnostics, USA). $\mathrm{C}$ reactive protein is analysed with a latex-enhanced immuno-turbidimetric test (Atellica CH 930, Siemens, Germany).

\section{Androgen treatment response}

Serum total testosterone, androstenedione, dehydroepiandrosterone sulfate and 17-hydroxyprogesterone are measured by liquid chromatography-tandem mass spectrometry (Acquity UPLC Xevo TQ MS, Waters, USA). Sex hormone-binding globulin is analysed by a competitive chemiluminescence based immunoassay (Cobas, Roche Diagnostics, Mannheim, Germany). Plasma luteinising hormone and follicle-stimulating hormone are measured using sandwich chemiluminescence immunoassay (ADVIA Centaur, Siemens, Germany).

\section{Additional measurements}

\section{Common terminology criteria for adverse events}

Adverse events will be registered at the 12-week postintervention visit, using the common terminology criteria for adverse events V.4. ${ }^{20}$

\section{Metabolic biomarkers}

Samples of full blood and serum are prospectively collected at baseline and 12-week postintervention for future assessment of cardiac, adipose and inflammatory biomarkers.

\section{Genetic biomarkers}

A biobank is generated during the trial and will be used for a future prospective, observational study assessing the predictive and prognostic value of genetic biomarkers in circulating cell-free DNA (ccfDNA). Samples from blood and urine are prospectively collected at baseline and at time of disease progression on allocated treatment. Somatic alterations will be analysed from ccfDNA in plasma, urine pellets and supernatant.

\section{Patient and public involvement}

The patients treated with enzalutamide and AAP inspired us to the design of the trial's research question and outcomes, by sharing their experience of the treatment and associated side effects in the out-patient clinic of Herlev and Gentofte Hospital. Fatigue is the primary outcome of the trial, since fatigue is the most common and distressing symptom experienced by patients with mCRPC. ${ }^{21}$ The burden of the intervention is partly assessed by patient-reported questionnaires assessing fatigue and HRQoL. Patients were not involved in the recruitment to or conduct of the trial. The results of the trial will be made publicly available through the homepage of Herlev and Gentofte Hospital.

\section{Sample size and statistical analysis}

The sample size calculation is based on the detection of a between-group MCID of 3.0 points on the FACIT-Fatigue scale, with an anticipated drop-out of $10 \% .{ }^{14}$ The standard deviation is assumed to be 6.55 , based on confidence limits from previous studies assessing fatigue in men with metastatic prostate cancer. ${ }^{22-24}$ The sample size calculation is based on a two-tailed significance level of $5 \%$ and a power of $80 \%$. This required a sample size of 85 participants in each group, a total of 170 men.

The within-subject and between-group differences of the primary and secondary endpoints will be analysed with linear mixed effect models using constrained longitudinal analysis (cLDA). The between-group MCID in fatigue and HRQoL will be analysed with risk difference. An MCID in fatigue is defined as an individual 3-point change in the FACIT-Fatigue total score. An MCID in HRQoL is defined as an individual 6-point change in FACT-P total score. Interactions between patient-reported outcomes (fatigue and quality of life), and age ( $<75$ vs $\geq 75$ years) and extent of metastases (high vs low volume disease) will be tested in sub-group analyses using forest plots. High volume disease is defined as $\geq 4$ bone metastases with $\geq 1$ bone metastases outside pelvis and column. Interactions between metabolic changes, and body mass index (BMI) $(<30$ vs $\geq 30)$ and age ( $<75$ vs $\geq 75$ years $)$ will be analysed in subgroup analyses using forest plots. The linear mixed effect model using cLDA handles random missing data.

\section{ETHICS AND DISSEMINATION}

Participants will receive standard first-line treatment for mCRPC. The primary investigator obtains the written informed consent from all participants. The trial follows the International Conference on Harmonization Good Clinical Practice guidelines, the latest revision of the Declaration of Helsinki and the Danish rules on Clinical Trials of Medicines in Humans. The trial is externally monitored by Good Clinical Practice Unit, Copenhagen University. The trial's results will be published in peer-reviewed international journals or otherwise made publicly available and will be presented at national and international conferences and symposiums irrespective of the outcomes. Patient-reported outcome, metabolic changes, hormone analyses and genetic biomarkers will 
be reported in separate publications. Study completion is expected by spring 2020, and dissemination of the results will begin as soon as possible thereafter.

\section{DISCUSSION}

This article describes the protocol of an ongoing randomised clinical trial comparing fatigue, HRQoL and metabolic changes in men with mCRPC treated with firstline enzalutamide versus AAP.

We chose fatigue as the primary endpoint since it is the most common and distressing symptom affecting HRQoL in men with mCRPC. ${ }^{352526}$ We assess changes in patient-reported fatigue with the validated 13-question questionnaire FACIT-Fatigue. Previous randomised clinical trials on enzalutamide and AAP measured the level of fatigue with Common Terminology Criteria for Adverse Event with a coarse three-level grading, from fatigue 'relieved by rest' to fatigue 'limiting self-care'. ${ }^{35}$ We expect that changes in fatigue will be reported more accurately from patients using the 13-question questionnaires in contrast to the physician reported three-level grading used in previously trials. We did not choose to assess fatigue by interviews since that would preclude a statistical comparison of changes in fatigue, even though interviews might yield a more individual assessment of fatigue.

We chose to assess changes in patient-reported HRQoL with the 39-question questionnaire FACT-P, because FACT-P is developed and validated for assessing HRQoL in men with prostate cancer. Changed HRQoL for men with metastatic prostate cancer has previously been measured with FACT-P in randomised clinical trials, and the results can therefore be compared with existing literature. ${ }^{27-29}$

We chose to comprehensively assess metabolic changes, including glucose metabolism measured with OGTT and HbAlc, and body composition measured with DXA scans. Previous randomised clinical trials on enzalutamide and abiraterone have measured following metabolic adverse events: plasma glucose, weight and blood pressure. ${ }^{3-6} \mathrm{We}$ did not choose to measure non-fasting plasma glucose, because the within-subject plasma glucose varies widely, and plasma glucose alone fails to diagnose $\sim 30 \%$ of cases of previously undiagnosed diabetes. ${ }^{30} 31$ We chose to assess glucose metabolism with OGTT and HbAlc because the hyperglycaemic disease process is a risk factor for microvascular complications, diabetes and cardiovascular disease and may be present without fulfilling the criteria for diabetes. ${ }^{30}$ We chose to measure BMI and body composition with DXA scans because both methods can identify obesity and associated metabolic and cardiovascular risks, ${ }^{32-34}$ while DXA scans can identify body fat which may be a better predictor of metabolic syndrome than BMI alone. ${ }^{35}{ }^{36}$ We measure LBM with DXA scans, because a loss of lean mass can over time contribute to a decrease in muscle strength which are important predictors of balance, the occurrence of falls and mortality. ${ }^{37} 38$
We chose 3-month follow-up to evaluate the treatments side effects and at the same time to avoid a pronounced influence of disease progression on HRQoL and fatigue. In a cohort study of 21 participants metabolic changes appeared already after $7-10$ days of treatment with low-dose prednisolone $(6 \mathrm{mg} /$ day $) .{ }^{39}$ Changes in fatigue and HRQoL can be experienced within the first 3 months of treatment with new androgen pathway inhibitors. ${ }^{27}$ The median time until biochemical progression was 11.1 and 11.2 months for men with mCRPC treated with AAP and enzalutamide, respectively. ${ }^{35}$ However, $14 \%(74 / 546)$ had biochemical progression after only 3 months' treatment with AAP; and $8 \%$ (70/854) had biochemical progression after 3 months' treatment with enzalutamide. ${ }^{35}$

The lack of blinding can be perceived as a weakness, but we find that it is of minor importance and unlikely to affect the objectively measured outcomes. Blood samples are analysed in an independent laboratory, DXA scans are analysed with the same software and outcomes on fatigue and HRQoL are reported by participants.

\section{CONCLUSION}

The aim of this trial is to assess differences in the patient-reported and metabolic side effects of enzalutamide and AAP. The results may in the future help patients and physicians to choose the best tolerated treatment and thereby reduce treatment induced morbidity and improve quality of life.

\section{Author affiliations \\ ${ }^{1}$ Department of Urology, Herlev and Gentofte Hospital, Herlev, Denmark ${ }^{2}$ Department of Oncology, Herlev and Gentofte Hospital, Herlev, Denmark ${ }^{3}$ Department of Endocrinology, Copenhagen University Hospital, Rigshospitalet, Copenhagen, Denmark \\ ${ }^{4}$ Department of Urology, Michigan Medicine, Ann Arbor, Michigan, USA}

Acknowledgements We want to thank the patients, treated with enzalutamide and AAP at Herlev and Gentofte Hospital, for inspiring us to design this trial by openly sharing their experience of their treatment and associated side effects.

Contributors KKT, PBØ, JS, CK, HL, MF, GP and RB participated in the design of the study. KKTconceived the study and participated in its design and coordination. KKT and $\mathrm{PB} \emptyset$ drafted the study protocol. KKT drafted the manuscript and all authors read and approved the final manuscript.

Funding JS, trial sponsor, PhD, DMSci, Chair Professor of Urology Mail: Jens. Soenksen@regionh.dk. This work was supported by SPCG Clinical Research Grant 2017 and the internal research fund of Herlev and Gentofte Hospital.

Competing interests $\mathrm{PB} \emptyset$ is speaker for Astellas, Ipsen and Ferring. MF is speaker for Ferring and Astellas and advisory board representative for Astellas.HL is advisory board representative for Roche, Janssen, Astellas, Bayer and Sanofi-Aventis.

Patient consent for publication Not required.

Ethics approval This trial is approved by the National Committee on Health Research Ethics (H-17001347), Danish Data Protection Agency (2012-58-0004) and Danish Medicines Agency (EudraCT no.: 2017-000027-99, www.clinicaltrialsre gister.eu).

Provenance and peer review Not commissioned; externally peer reviewed. Data availability statement There are no data in this work.

Open access This is an open access article distributed in accordance with the Creative Commons Attribution Non Commercial (CC BY-NC 4.0) license, which permits others to distribute, remix, adapt, build upon this work non-commercially, and license their derivative works on different terms, provided the original work is 
properly cited, appropriate credit is given, any changes made indicated, and the use is non-commercial. See: http://creativecommons.org/licenses/by-nc/4.0/.

\section{REFERENCES}

1. Potter GA, Barrie SE, Jarman M, et al. Novel steroidal inhibitors of human cytochrome P45017.alpha.-Hydroxylase-C17,20-lyase): potential agents for the treatment of prostatic cancer. J Med Chem 1995;38:2463-71.

2. Auchus RJ, Yu MK, Nguyen S, et al. Use of prednisone with abiraterone acetate in metastatic castration-resistant prostate cancer. Oncologist 2014;19:1231-40.

3. Ryan CJ, Smith MR, de Bono JS, et al. Abiraterone in metastatic prostate cancer without previous chemotherapy. N Engl J Med 2013;368:138-48.

4. Rathkopf DE, Smith MR, de Bono JS, et al. Updated interim efficacy analysis and long-term safety of abiraterone acetate in metastatic castration-resistant prostate cancer patients without prior chemotherapy (COU-AA-302). Eur Urol 2014;66:815-25

5. Beer TM, Armstrong AJ, Rathkopf DE, et al. Enzalutamide in metastatic prostate cancer before chemotherapy. N Engl J Med 2014;371:424-33.

6. Evans CP, Higano CS, Keane T, et al. The prevail study: primary outcomes by site and extent of baseline disease for Enzalutamidetreated men with Chemotherapy-naïve metastatic castrationresistant prostate cancer. Eur Urol 2016;70:675-83.

7. Storey DJ, McLaren DB, Atkinson MA, et al. Clinically relevant fatigue in men with hormonesensitive prostate cancer on long-term androgen deprivation therapy. Ann Oncol 2012.

8. Østergren PB, Kistorp C, Bennedbæk FN, et al. The use of exercise interventions to overcome adverse effects of androgen deprivation therapy. Nat Rev Urol 2016;13:353-64.

9. Moreira RB, Debiasi M, Francini E, et al. Differential side effects profile in MCRPC patients treated with abiraterone or enzalutamide: a meta-analysis of randomized controlled trials. Clin Adv Hematol Oncol 2016;14(4 Suppl 5):14-15.

10. Davis ID, Martin AJ, Stockler MR, et al. Enzalutamide with standard first-line therapy in metastatic prostate cancer. $N$ Engl J Med 2019;381:121-31.

11. James ND, de Bono JS, Spears MR, et al. Abiraterone for prostate cancer not previously treated with hormone therapy. $N$ Engl J Med 2017;377:338-51.

12. Fizazi K, Tran N, Fein L, et al. Abiraterone plus prednisone in metastatic, Castration-Sensitive prostate cancer. N Engl J Med 2017;377:352-60.

13. Scher HI, Morris MJ, Stadler WM, et al. Trial design and objectives for castration-resistant prostate cancer: updated recommendations from the prostate cancer clinical trials Working group 3. J Clin Oncol 2016;34:1402-18.

14. Cella D, Eton DT, Lai J-S, et al. Combining anchor and distributionbased methods to derive minimal clinically important differences on the functional assessment of cancer therapy (fact) anemia and fatigue scales. J Pain Symptom Manage 2002;24:547-61.

15. Cella D, Nichol MB, Eton D, et al. Estimating clinically meaningful changes for the Functional Assessment of Cancer Therapy-Prostate: results from a clinical trial of patients with metastatic hormone-refractory prostate cancer. Value Health 2009;12:124-9.

16. Bredella MA, Gill CM, Keating LK, et al. Assessment of abdominal fat compartments using DXA in premenopausal women from anorexia nervosa to morbid obesity. Obesity 2013;21:2458-64.

17. Micklesfield LK, Goedecke JH, Punyanitya M, et al. Dual-Energy $\mathrm{X}$-ray performs as well as clinical computed tomography for the measurement of visceral fat. Obesity 2012;20:1109-14.

18. Matsuda M, DeFronzo RA. Insulin sensitivity indices obtained fro M oral glucose tolerance testing. Diabetes Care 1999;22.

19. Patarrão RS, Wayne Lautt W, Paula Macedo M. Assessment of methods and indexes of insulin sensitivity. Rev Port Endocrinol Diabetes e Metab 2014.
20. Services $\mathrm{H}$. Common terminology criteria for adverse events (CTCAE) version 4.0, 2010.

21. Colloca G, Venturino A, Governato I, et al. Incidence and correlates of fatigue in metastatic castration-resistant prostate cancer: a systematic review. Clin Genitourin Cancer 2016;14:5-11.

22. Cormie P, Galvão DA, Spry N, et al. Can supervised exercise prevent treatment toxicity in patients with prostate cancer initiating androgen-deprivation therapy: a randomised controlled trial. BJU Int 2015;115:256-66.

23. Bourke L, Gilbert S, Hooper R, et al. Lifestyle changes for improving disease-specific quality of life in sedentary men on long-term androgen-deprivation therapy for advanced prostate cancer: a randomised controlled trial. Eur Urol 2014;65:865-72.

24. Bourke L, Doll H, Crank $\mathrm{H}$, et al. Lifestyle intervention in men with advanced prostate cancer receiving androgen suppression therapy: a feasibility study. Cancer Epidemiol Biomarkers Prev 2011;20:647-57.

25. Slovin S, Clark W, Carles J, et al. Seizure rates in EnzalutamideTreated men with metastatic castration-resistant prostate cancer and risk of seizure. JAMA Oncol 2018;4.

26. Salem S, Komisarenko M, Timilshina N, et al. Impact of abiraterone acetate and enzalutamide on symptom burden of patients with Chemotherapy-naive metastatic castration-resistant prostate cancer. Clin Oncol 2017;29:601-8.

27. Khalaf DJ, Sunderland K, Eigl BJ, et al. Health-Related quality of life for abiraterone plus prednisone versus enzalutamide in patients with metastatic castration-resistant prostate cancer: results from a phase II randomized trial. Eur Urol 2019;75:940-7.

28. Basch E, Autio K, Ryan CJ, et al. Abiraterone acetate plus prednisone versus prednisone alone in chemotherapy-naive men with metastatic castration-resistant prostate cancer: patientreported outcome results of a randomised phase 3 trial. Lancet Oncol 2013.

29. Loriot $\mathrm{Y}$, Miller K, Sternberg CN, et al. Effect of enzalutamide on health-related quality of life, pain, and skeletal-related events in asymptomatic and minimally symptomatic, chemotherapy-naive patients with metastatic castration-resistant prostate cancer (prevail): results from a randomised, P. Lancet Oncol 2015.

30. Diabetes DOF, American Diabetes Association. Diagnosis and classification of diabetes mellitus. Diabetes Care 2009.

31. World Health Organisation (WHO), International Diabetes Federation (IDF). Definition and diagnosis of diabetes mellitus and intermediate hyperglycemia. World Heal Organ, 2006.

32. World Heal Organ - Tech Rep Ser. Obesity: preventing and managing the global epidemic. Report of a who consultation, 2000.

33. Lang PO, Trivalle C, Vogel T, et al. Markers of metabolic and cardiovascular health in adults: comparative analysis of DEXA-based body composition components and BMI categories. J Cardiol 2015.

34. Zhu S, Wang Z, Shen W, et al. Percentage body fat ranges associated with metabolic syndrome risk: results based on the third National health and nutrition examination survey (1988-1994). Am J Clin Nutr 2003;78:228-35.

35. Liu P, Ma F, Lou H, et al. The utility of fat mass index vs. body mass index and percentage of body fat in the screening of metabolic syndrome. BMC Public Health 2013;13.

36. Gómez-Ambrosi J, Silva C, Galofré JC, et al. Body mass index classification misses subjects with increased cardiometabolic risk factors related to elevated adiposity. Int J Obes 2012.

37. Lee CG, Boyko EJ, Nielson CM, et al. Mortality risk in older men associated with changes in weight, lean mass, and fat mass. J Am Geriatr Soc 2011.

38. Wolfson L, Judge J, Whipple R, et al. Strength is a major factor in balance, gait, and the occurrence of falls. J Gerontol A Biol Sci Med Sci 1995.

39. Petersons CJ, Mangelsdorf BL, Jenkins AB, et al. Effects of low-dose prednisolone on hepatic and peripheral insulin sensitivity, insulin secretion, and abdominal adiposity in patients with inflammatory rheumatologic disease. Diabetes Care 2013;36:2822-9. 